\title{
Spectral analysis of singular Sturm-Liouville operators on time scales
}

\begin{abstract}
In this paper, we consider properties of the spectrum of a SturmLiouville operator on time scales. We will prove that the regular symmetric Sturm-Liouville operator is semi-bounded from below. We will also give some conditions for the self-adjoint operator associated with the singular SturmLiouville expression to have a discrete spectrum. Finally, we will investigate the continuous spectrum of this operator.
\end{abstract}

1. Introduction. A time scale $\mathbb{T}$ is an arbitrary nonempty closed set of real numbers. Dynamic equations on time scales has attracted much interest because it unites the theory of differential and difference equations. It has led to several important applications, e.g., in the study of heat transfer, insect population models, epidemic models stock market, and neural networks (see [13], [17], [24], [25]). However, there are very few results known for the Sturm-Liouville operators on time scales.

On the other hand, the spectral analysis of a self-adjoint differential operator is one of the most popular problems in operator theory. The spectrum of such operators depends on the behavior of the coefficients of the corresponding differential expression. This problem has been investigated by many mathematicians (see [4], [8]-[10], [14], [16], [20], [22], [26]).

2010 Mathematics Subject Classification. 34N05, 47A10, 47B25.

Key words and phrases. Sturm-Liouville operator, time scales, splitting method, discrete spectrum, continuous spectrum. 
The aim of this paper is to extend some results for differential operators obtained in [10] to the case of Sturm-Liouville dynamic equation

$$
L(y):=-\left[p(t) y^{\Delta}(t)\right]^{\nabla}+q(t) y(t)=\lambda y(t), t \in[a, \infty)_{\mathbb{T}},
$$

where $p, q$ are real-valued continuous functions on $\mathbb{T}$ and $p(t) \neq 0$ for all $t \in \mathbb{T}$. We prove that the regular symmetric Sturm-Liouville operator is semi-bounded from below. Using the splitting method [10], we will give some conditions for the self-adjoint operator associated with the singular expression (1) to have a discrete spectrum. We also investigate the continuous spectrum of this operator.

2. Preliminaries. Now, we recall some necessary fundamental concepts of time scales, and we refer to [3], [5], [6], [11], [13], [15], [19] for more details.

Definition 1. Let $\mathbb{T}$ be a time scale. The forward jump operator $\sigma: \mathbb{T} \rightarrow \mathbb{T}$ is defined by

$$
\sigma(t)=\inf \{s \in \mathbb{T}: s>t\}, t \in \mathbb{T}
$$

and the backward jump operator $\rho: \mathbb{T} \rightarrow \mathbb{T}$ is defined by

$$
\rho(t)=\sup \{s \in \mathbb{T}: s<t\}, t \in \mathbb{T} .
$$

It is convenient to consider the graininess operators $\mu_{\sigma}: \mathbb{T} \rightarrow[0, \infty)$ and $\mu_{\rho}: \mathbb{T} \rightarrow(-\infty, 0]$ defined by $\mu_{\sigma}(t)=\sigma(t)-t$ and $\mu_{\rho}(t)=\rho(t)-t$, respectively. A point $t \in \mathbb{T}$ is left scattered if $\mu_{\rho}(t) \neq 0$ and left dense if $\mu_{\rho}(t)=0$. A point $t \in \mathbb{T}$ is right scattered if $\mu_{\sigma}(t) \neq 0$ and right dense if $\mu_{\sigma}(t)=0$. We introduce the sets $\mathbb{T}^{k}, \mathbb{T}_{k}, \mathbb{T}^{*}$ which are derived from the time scale $\mathbb{T}$ as follows. If $\mathbb{T}$ has a left scattered maximum $t_{1}$, then $\mathbb{T}^{k}=\mathbb{T}-\left\{t_{1}\right\}$, otherwise $\mathbb{T}^{k}=\mathbb{T}$. If $\mathbb{T}$ has a right scattered minimum $t_{2}$, then $\mathbb{T}_{k}=\mathbb{T}-\left\{t_{2}\right\}$, otherwise $\mathbb{T}_{k}=\mathbb{T}$. Finally, $\mathbb{T}^{*}=\mathbb{T}^{k} \cap \mathbb{T}_{k}$.

Definition 2. A function $f$ on $\mathbb{T}$ is said to be $\Delta$-differentiable at some point $t \in \mathbb{T}$ if there is a number $f^{\Delta}(t)$ such that for every $\varepsilon>0$ there is a neighborhood $U \subset \mathbb{T}$ of $t$ such that

$$
\left|f(\sigma(t))-f(s)-f^{\Delta}(t)(\sigma(t)-s)\right| \leq \varepsilon|\sigma(t)-s|, s \in U .
$$

Analogously one may define the notion of a $\nabla$-differentiability of some function using the backward jump $\rho$. One can show (see [5])

$$
f^{\Delta}(t)=f^{\nabla}(\sigma(t)), \quad f^{\nabla}(t)=f^{\Delta}(\rho(t))
$$

for continuously differentiable functions.

Example 3. If $\mathbb{T}=\mathbb{R}$, then we have

$$
\sigma(t)=t, f^{\Delta}(t)=f^{\prime}(t) .
$$

If $\mathbb{T}=\mathbb{Z}$, then we have

$$
\sigma(t)=t+1, f^{\Delta}(t)=\Delta f(t)=f(t+1)-f(t) .
$$


If $\mathbb{T}=q^{\mathbb{N}_{0}}=\left\{q^{k}: q>1, k \in \mathbb{N}_{0}\right\}$, then we have

$$
\sigma(t)=q t, f^{\Delta}(t)=\frac{f(q t)-f(t)}{q t-t} .
$$

Definition 4. Let $f: \mathbb{T} \rightarrow \mathbb{R}$ be a function, and $a, b \in \mathbb{T}$. If there exists a function $F: \mathbb{T} \rightarrow \mathbb{R}$, such that $F^{\Delta}(t)=f(t)$ for all $t \in \mathbb{T}^{k}$, then $F$ is a $\Delta$-antiderivative of $f$. In this case the integral is given by the formula

$$
\int_{a}^{b} f(t) \Delta t=F(b)-F(a) \text { for } a, b \in \mathbb{T} .
$$

Analogously one may define the notion of $\nabla$-antiderivative of some function.

Let $L_{\nabla}^{2}(\mathbb{T})$ be the space of all functions defined on $\mathbb{T}$ such that

$$
\|f\|:=\left(\int_{a}^{b}|f(t)|^{2} \nabla t\right)^{1 / 2}<\infty .
$$

Let $\mathbb{T}$ be a time scale which is bounded from below and unbounded from above such that $\inf \mathbb{T}=a>-\infty$ and $\sup \mathbb{T}=\infty$. We will denote $\mathbb{T}$ also as $[a, \infty)_{\mathbb{T}}$.

The space $L_{\nabla}^{2}[a, \infty)_{\mathbb{T}}$ is a Hilbert space with the inner product (see [23])

$$
(f, g):=\int_{a}^{\infty} f(t) \overline{g(t)} \nabla t, \quad f, g \in L_{\nabla}^{2}[a, \infty)_{\mathbb{T}} .
$$

The Wronskian of $y(),. z($.$) is defined by (see [5])$

$$
W_{t}(y, z):=p(t)\left[y(t) z^{\Delta}(t)-y^{\Delta}(t) z(t)\right], t \in \mathbb{T} .
$$

Definition 5. Let $D_{A}$ denote a subset of the complex Hilbert space $H$. A linear operator $A$ is said to be Hermitian if, for all $x, y \in D_{A},(A x, y)=$ $(x, A y)$ holds. A Hermitian operator with a domain of definition dense in $H$ is called a symmetric operator. An operator $A^{*}$ defined on $H$ is called the adjoint of symmetric operator $A$ if for all $x, y \in D_{A},(x, A y)=\left(A^{*} x, y\right)$. An operator with a domain of definition dense in $H$ is said to be self-adjoint if $A=A^{*}$. An operator $A$ is said to be compact if it maps every bounded set into a compact set (see [21]).

Definition 6. A complex number $\lambda$ is called a regular point of the linear operator $A$ acting in complex Hilbert space $H$ if

(R1) the inverse $R_{\lambda}(A)=(A-\lambda I)^{-1}$ (where $I$ is the identity operator in $H)$ exists, and

(R2) $R_{\lambda}(A)$ is a bounded operator defined on the whole space $H$.

Assume that

(R3) $R_{\lambda}(A)$ is defined on a set which is dense in $H$.

The operator $R_{\lambda}(A)$ is then called the resolvent of the operator $A$. All non-regular points $\lambda$ are called points of the spectrum of the operator $A$. 
The point spectrum or discrete spectrum $\sigma_{p}(A)$ is the set such that $R_{\lambda}(A)$ does not exist. A number $\lambda \in \sigma_{p}(A)$ is called an eigenvalue of $A$. The spectrum of the operator $A$ is said to be purely discrete if it consists of a denumerable set of eigenvalues with no finite point of accumulation.

The continuous spectrum $\sigma_{c}(A)$ is the set such that $R_{\lambda}(A)$ exists and satisfies (R3) but not (R2).

The residual spectrum $\sigma_{r}(A)$ is the set such that $R_{\lambda}(A)$ exists but does not satisfy (R3) (see [18]).

Theorem 7 ([18]). The residual spectrum $\sigma_{r}(A)$ of a self-adjoint linear operator acting on a complex Hilbert space $H$ is empty.

Theorem 8 ([21]). All self-adjoint extensions of a closed, symmetric operator which has equal and finite deficiency indices have one and the same continuous spectrum.

Definition 9 ([21]). The direct sum $A_{1} \oplus A_{2}$ of two operators $A_{1}, A_{2}$ in the spaces $H_{1}, H_{2}$ is an operator in the space $H_{1} \oplus H_{2}$ of all ordered pairs $\left\{x_{1}, x_{2}\right\}, x_{1} \in H_{1}, x_{2} \in H_{2}$; its domain of definition is the set of all ordered pairs $\left\{x_{1}, x_{2}\right\}, x_{1} \in D_{A_{1}}, x_{2} \in D_{A_{2}}$, and

$$
\left(A_{1} \oplus A_{2}\right)\left\{x_{1}, x_{2}\right\}=\left\{A_{1} x_{1}, A_{2} x_{2}\right\} .
$$

It is easily seen that if $A_{1}$ and $A_{2}$ are each self-adjoint operators, then their direct sum $A_{1} \oplus A_{2}$ is also a self-adjoint operator.

Definition $10([21])$. A symmetric operator $A$ is said to be semi-bounded from below if there is a number $m$ such that, for all $x \in D_{A}$, the inequality

$$
(A x, x) \geq m\|x\|^{2}
$$

holds. Similarly, if there is a number $M$ such that for all $x \in D_{A}$, the inequality

$$
(A x, x) \leq M\|x\|^{2}
$$

holds, then $A$ is said to be semi-bounded from above.

Theorem 11 ([21]). If a symmetric operator A with finite deficiency indices $(n, n)$ satisfies the condition

$$
(A x, x) \geq m\|x\|^{2}, \quad x \in D_{A},
$$

or the condition

$$
(A x, x) \leq M\|x\|^{2}, \quad x \in D_{A},
$$

then the part of the spectrum of every self-adjoint extension of $A$ which lies to the left of $m$ or to the right of $M$ can consist of only a finite number of eigenvalues and the sum of their multiplicities does not exceed $n$. 
3. Main Results. Let us consider the linear set $D_{\max }$ consisting of all vectors $y \in L_{\nabla}^{2}[a, \infty)_{\mathbb{T}}$ such that $y$ and $p y^{\nabla}$ are locally $\Delta$ absolutely continuous functions on $[a, \infty)_{\mathbb{T}}$ and $L y \in L_{\nabla}^{2}[a, \infty)_{\mathbb{T}}$. We define the maximal operator $L_{\max }$ on $D_{\max }$ by the equality $L_{\max } y=L y$.

For every $y, z \in D_{\max }$ we have Green's formula

$$
(L y, z)-(y, L z)=[y, z]_{b}-[y, z]_{a}, b \in[a, \infty)_{\mathbb{T}}
$$

where $[y, z]_{t}:=p(t)\left\{y(t) z^{\nabla(t)}-y^{\nabla}(t) \overline{z(t)}\right\}$ (see [11]).

Let $D_{\min }$ be the linear set of all vectors $y \in D_{\max }$ satisfying the conditions

$$
y(a)=\left(p y^{\nabla}\right)(a)=y(b)=\left(p y^{\nabla}\right)(b)=0, b \in(a, \infty)_{\mathbb{T}} .
$$

The operator $L_{\min }$, that is the restriction of the operator $L_{\max }$ to $D_{\min }$ is called the minimal operator and the equalities $L_{\max }=L_{\min }^{*}$ holds. Further (it follows from (3)), $L_{\min }$ is a closed symmetric operator with deficiency indices $(2,2)([7],[21])$.

Theorem 12. If $p(t)>0$ ( $\left.t \in[a, b]_{\mathbb{T}}, a<b<\infty\right)$, then the regular operator $L_{\min }$ is semi-bounded from below. Further, the negative part of the spectrum of every self-adjoint extension of $L_{\min }$ consists of not more that a finite number of negative eigenvalues of finite multiplicity.

Proof. For $y \in D_{\min }$ we have

$$
y(a)=\left(p y^{\nabla}\right)(a)=y(b)=\left(p y^{\nabla}\right)(b)=0 .
$$

By integration by parts, we get

$$
\begin{aligned}
\left(L_{\min } y, y\right) & =\int_{a}^{b} L y \bar{y} \nabla t=\int_{a}^{b}\left[-\left[p(t) y^{\Delta}(t)\right]^{\nabla}+q(t) y(t)\right] \bar{y} \nabla t \\
& =\int_{a}^{b}\left[-\left[p(t) y^{\Delta}(t)\right]^{\nabla} \bar{y}+q(t)|y|^{2}\right] \nabla t \\
& =\int_{a}^{b}\left[p(t)\left|y^{\Delta}\right|^{2}+q(t)|y|^{2}\right] \nabla t .
\end{aligned}
$$

We set

$$
v(t, \xi)= \begin{cases}1, & \xi \leq t \\ 0, & \xi>t\end{cases}
$$

and

$$
H(\xi, \eta)=-\int_{a}^{b} q(t) v(t, \xi) v(t, \eta) \nabla t .
$$

For $y \in D_{\min }$ we have

$$
y(t)=\int_{a}^{b} \frac{v(t, \xi)\left(p y^{\Delta}\right)(\xi)}{p(\xi)} \nabla \xi
$$


Hence we get

$$
\begin{aligned}
& \left(L_{\min } y, y\right)=\int_{a}^{b} \frac{\left|\left(p y^{\Delta}\right)(\xi)\right|^{2}}{p(\xi)} \nabla \xi \\
& -\int_{a}^{b} \int_{a}^{b} \frac{\left.H(\xi, \eta)\left(p y^{\Delta}\right)(\xi)\left(p \bar{y}^{\Delta}\right)(\eta)\right)}{p(\xi) p(\eta)} \nabla \xi \nabla \eta .
\end{aligned}
$$

Let $L_{\nabla, p}^{2}(a, b)$ be the Hilbert space of all complex-valued functions defined on $[a, b]$ with the inner product

$$
\left(f_{1}, f_{2}\right)_{1}=\int_{a}^{b} f_{1}(t) \overline{f_{2}(t)} \frac{1}{p(t)} \nabla t .
$$

In $L_{\nabla, p}^{2}(a, b)$ we consider the integral operator $K$ with the symmetric kernel $H(\xi, \eta)$ :

where

$$
K f=\int_{a}^{b} \frac{H(\xi, \eta)}{p(\eta)} f(\eta) \nabla \eta
$$

$$
\int_{a}^{b} \int_{a}^{b} \frac{|H(\xi, \eta)|^{2}}{p(\xi) p(\eta)} \nabla \xi \nabla \eta<\infty
$$

Then $K$ is a compact operator in the space $L_{\nabla, p}^{2}(a, b)$.

Let $\varphi_{1}, \varphi_{2}, \varphi_{3}, \ldots$ be a complete orthonormal system of eigenfunctions of the operator $K$ and $\lambda_{1}, \lambda_{2}, \lambda_{3}, \ldots$ be the corresponding eigenvalues. Then we get

$$
(K f, f)_{1}=\sum_{k=1}^{\infty} \lambda_{k}\left|\left(f, \varphi_{k}\right)_{1}\right|^{2} .
$$

As $k \rightarrow \infty$, we have $\lambda_{k} \rightarrow 0$. Then there is a certain number $N$ such that $\lambda_{k}<1$ for $k>N$. For $\left(f, \varphi_{k}\right)_{1}=0, k=1,2, \ldots, N$, we have

$$
(K f, f)_{1}=\sum_{k=N+1}^{\infty} \lambda_{k}\left|\left(f, \varphi_{k}\right)_{1}\right|^{2} \leq \sum_{k=N+1}^{\infty}\left|\left(f, \varphi_{k}\right)_{1}\right|^{2},
$$

that is,

$$
(K f, f)_{1} \leq(f, f)_{1} .
$$

Let $\mathcal{D}$ denote the manifold of all functions $y \in D_{\text {min }}$ which satisfy the conditions

$$
\left(p y^{\Delta}, \varphi_{k}\right)_{1}=0, k=1,2, \ldots, N, y \in D_{\min } .
$$

By (5), we have, for $y \in \mathcal{D}$,

$$
\begin{gathered}
\int_{a}^{b} \int_{a}^{b} \frac{\left.H(\xi, \eta)\left(p y^{\Delta}\right)(\xi)\right)\left(p \bar{y}^{\Delta}(\eta)\right)}{p(\xi) p(\eta)} \nabla \xi \nabla \eta \leq\left(K p y^{\Delta}, p y^{\Delta}\right)_{1} \\
\leq\left(p y^{\Delta}, p y^{\Delta}\right)_{1}=\int_{a}^{b} \frac{\left|\left(p y^{\Delta}\right)(\xi)\right|^{2}}{p(\xi)} \nabla \xi .
\end{gathered}
$$


From the equality (4), we obtain

$$
\left(L_{\min } y, y\right) \geq 0 .
$$

On the other hand, the dimension of the manifold $D_{\min }$ modulo $\mathcal{D}$ is $N$, and consequently, the operator $L_{\text {min }}$ is semi-bounded from below on the whole manifold $D_{\text {min }}$. By Theorem 11, we get the desired result.

Let $H^{\prime}$ denote the set of all functions $f$ from $L_{\nabla}^{2}(0, \infty)_{\mathbb{T}}$ which vanish outside a finite interval $[\alpha, \beta] \subset[0, \infty)_{\mathbb{T}}$ and $D_{\min }^{\prime}=H^{\prime} \cap D_{\min }$.

Further, let $L_{\min }^{\prime}$ denote the restriction of the operator $L_{\min }$ to $D_{\min }^{\prime}$. Then $L_{\min }$ is the closure of the operator $L_{\min }^{\prime}$, i.e., $\widetilde{L_{\min }^{\prime}}=L_{\min }([21])$.

Now we restrict $D_{\min }^{\prime}$ by imposing the additional conditions

$$
y(c)=\left(p y^{\nabla}\right)(c)=0,
$$

where $c$ is a fixed point of the interval $(a, \infty)_{\mathbb{T}}$. By this restriction, we obtain the manifold $D_{\min }^{\prime \prime}$.

The restriction $L_{\min }^{\prime \prime}$ of the operator $L_{\min }^{\prime}$ to $D_{\min }^{\prime \prime}$ is called the splitting of the operator $L_{\min }^{\prime}$ at the point $c$ of the interval $(a, \infty)_{\mathbb{T}}$. It is clear that

$$
L_{\min }^{\prime \prime}=L_{1}^{\prime} \oplus L_{2}^{\prime}
$$

i.e., the operator $L_{\min }^{\prime \prime}$ is the direct sum of two operators $L_{1}^{\prime}$ and $L_{2}^{\prime}$ in the spaces $L_{\nabla}^{2}(0, c)_{\mathbb{T}}$ and $L_{\nabla}^{2}(c, \infty)_{\mathbb{T}}$, where $L_{1}^{\prime}$ and $L_{2}^{\prime}$ are generated in these spaces from the Sturm-Liouville expression $L$ in the same way as $L_{\min }^{\prime}$ was.

If $L_{1}=\widetilde{L_{1}^{\prime}}$ and $L_{2}=\widetilde{L_{2}^{\prime}}$ are the closures of the operators $L_{1}^{\prime}$ and $L_{2}^{\prime}$, then (6) implies that

$$
\widetilde{L_{\min }^{\prime \prime}}=L_{1} \oplus L_{2} \text {. }
$$

If we extend the symmetric operators $L_{1}$ and $L_{2}$ into self-adjoint operators $L_{1, s}$ and $L_{2, s}$ in the spaces $L_{\nabla}^{2}(0, c)_{\mathbb{T}}$ and $L_{\nabla}^{2}(c, \infty)_{\mathbb{T}}$ respectively, then the direct sum

$$
A=L_{1, s} \oplus L_{2, s}
$$

will be a self-adjoint extension of the symmetric operator $\widetilde{L_{\text {min }}^{\prime \prime}}$. The spectrum of the operator $A$ is the set-theoretic sum of the spectra of $L_{1, s}$ and $L_{2, s}$.

Since the deficiency indices of the operator $\widetilde{L_{\text {min }}^{\prime \prime}}$ are finite, by Theorem 8 , all its self-adjoint extensions have one and the same continuous spectrum. Both the operator $A$ and also each self-adjoint extension $L_{s}$ of the operator $L_{\text {min }}$ are such extensions. Hence, the continuous parts of the spectrum of the two operators $A$ and $L_{s}$ coincide.

Therefore, we have the following theorem:

Theorem 13. The continuous parts of the spectrum of every self-adjoint extension of the operator $L_{\min }$ is the set-theoretic sum of the continuous parts of the spectra of $L_{1, s}$ and $L_{2, s}$, where $L_{1, s}$ and $L_{2, s}$ have been obtained by the splitting of the operator $L_{\min }$. 
Theorem 14. If

$$
\lim _{t \rightarrow \infty} q(t)=+\infty
$$

and

$$
p(t)>0, \quad t \in[a, \infty)_{\mathbb{T}}
$$

then every self-adjoint extension $L_{s}$ of the singular operator $L_{\min }$ has a purely discrete spectrum.

Proof. Let $N>0$ be an arbitrary number. From (7), one can choose a number $c$ such that

$$
|q(t)|>N \text { for } c<t<\infty .
$$

By the condition (8), via integration by parts, we obtain $\left(y \in D_{L_{2}^{\prime}}\right)$

$$
\begin{aligned}
\left(L_{2}^{\prime} y, y\right) & =\int_{c}^{\infty} L y \bar{y} \nabla t=\int_{c}^{\infty}\left[-\left[p(t) y^{\Delta}(t)\right]^{\nabla}+q(t) y(t)\right] \bar{y} \nabla t \\
& =\int_{c}^{\infty}\left[-\left[p(t) y^{\Delta}(t)\right]^{\nabla} \bar{y}+q(t)|y|^{2}\right] \nabla t \\
& =\int_{c}^{\infty} p\left|y^{\Delta}\right|^{2}+q(t)|y|^{2} \nabla t>N \int_{c}^{\infty}|y|^{2} \nabla t=N(y, y) .
\end{aligned}
$$

Hence the operator $L_{2}^{\prime}$ is bounded from below and its closure $L_{2}$ is also bounded from below by the number $N$. Therefore, by Theorem 11, the half-axis $-\infty<\lambda<N$, contains no point of the continuous spectrum of the self-adjoint extension $L_{2, s}$ of $L_{2}$.

On the other hand, since the operator $L_{1}$ is regular, the spectrum of any self-adjoint extension $L_{1, s}$ of $L_{1}$ is purely discrete. Hence the half-axis $-\infty<$ $\lambda<N$, contains no point of the continuous spectrum of $A=L_{1, s} \oplus L_{2, s}$.

By Theorem 13, every self-adjoint extension $L_{s}$ of the operator $L_{\min }$ has this property. Since the number $N$ is arbitrary, the spectrum of the operator $L_{s}$ has no continuous part at all.

Theorem 15. Let

$$
\lim _{t \rightarrow \infty} q(t)=M
$$

and $p(t)>0\left(t \in[0, \infty)_{\mathbf{T}}\right)$. Then the interval $(-\infty, M)$ contains no point of the continuous spectrum of any self-adjoint extension $L_{s}$ of the singular operator $L_{\min }$; on the contrary, any $L_{s}$ can only have at most point-eigenvalues on this interval and these can have a point of accumulation only at $\lambda=M$.

Proof. If we decompose the operator at a point $c$ such that

$$
q(t)>M-\varepsilon \text { for } c<t<\infty,
$$

then we obtain

$$
\left(L_{2}^{\prime} y, y\right)>(M-\varepsilon)(y, y) .
$$


Hence, the part of the spectrum of $L_{2}$ lying in the interval $(-\infty, M-\varepsilon)$ can consist only of a finite number of eigenvalues of finite multiplicity. On the other hand, by Theorem 12, the operator $L_{1}$ is regular and bounded below. Hence its spectrum is purely discrete and any point of accumulation of the spectrum $L_{1, s}$ can only be at $\lambda=+\infty$. Thus, from Theorem 13, we get the desired result.

Now, we need the following lemma.

Lemma 16. If the interval $\left[\lambda_{0}-\delta, \lambda_{0}+\delta\right]$ contains no point of the spectrum of a self-adjoint operator $A$ except perhaps for a finite number of eigenvalues, each of finite multiplicity, and if $Q$ is a bounded Hermitian operator satisfying the condition

$$
\|Q\|<\delta
$$

then the point $\lambda_{0}$ does not lie in the continuous part of the spectrum of the operator $A+Q$.

Proof. See [21].

Theorem 17. Let $p(t) \equiv 1$ and

$$
\lim _{t \rightarrow \infty}|q(t)|=M \text {. }
$$

Then any interval, of length greater than $2 M$, of the positive half-axis contains points of the continuous spectrum of any self-adjoint extension $L_{s}$ of the singular operator $L_{\min }$.

Proof. Suppose, contrary to our claim, that an interval $\left[\lambda_{0}-\delta, \lambda_{0}+\delta\right]$ of the half-axis $\lambda>0$ contains no point of the continuous spectrum of $L_{s}$, $\delta>M$. Then, though the operator may be decomposed, this interval would contain no point of the continuous spectrum of any self-adjoint extension of $L_{2}$. If we choose the point $c$ such that

$$
|q(t)| \leq M+\varepsilon<\delta \text { for } t>c,
$$

then, by Lemma $16, \lambda_{0}$ can not belong to the continuous spectrum of the self-adjoint extension of the minimal operator generated by the expression $-\left[p(t) y^{\Delta}(t)\right]^{\nabla}$ and the same boundary conditions. But this is contradiction because the continuous spectrum of the last operator covers the whole of the positive half-axis.

In particular, for $M=0$ we have the following corollary.

Corollary 18. Let $p(t) \equiv 1$ and

$$
\varlimsup_{t \rightarrow \infty}|q(t)|=0 .
$$

Then the whole positive half-axis is covered by the continuous spectrum of any self-adjoint extension $L_{s}$ of the singular operator $L_{\min }$. 
Corollary 19. Let $p(t) \equiv 1$ and

$$
\varlimsup_{t \rightarrow \infty}|q(t)|=\rho<\infty, \lim _{t \rightarrow \infty}|q(t)|=\sigma>-\infty .
$$

Then any interval, of length greater than $\rho-\sigma$, of the half-axis

$$
\lambda>\frac{1}{2}(\rho+\sigma)
$$

contains points of the continuous spectrum of any self-adjoint extension $L_{s}$ of the singular operator $L_{\min }$.

Proof. For, if $q_{1}(t)=q(t)-\frac{1}{2}(\rho+\sigma)$, then

$$
\varlimsup_{t \rightarrow \infty}\left|q_{1}(t)\right|=\frac{1}{2}(\rho-\sigma),
$$

and the result follows by replacing $q(t)$ by $q_{1}(t)$, i.e., by applying Theorem 17 to the operator $L_{s}-\frac{1}{2}(\rho+\sigma) I$.

\section{REFERENCES}

[1] Agarwal, R. P., Bohner, M., Li, W.-T., Nonoscillation and Oscillation Theory for Functional Differential Equations, Marcel Dekker, New York, 2004.

[2] Anderson, D. R., Guseinov, G. Sh., Hoffacker, J., Higher-order self-adjoint boundaryvalue problems on time scales, J. Comput. Appl. Math. 194 (2) (2006), 309-342.

[3] Atici Merdivenci, F., Guseinov, G. Sh., On Green's functions and positive solutions for boundary value problems on time scales, J. Comput. Appl. Math. 141 (1-2) (2002), 75-99.

[4] Berkowitz, J., On the discreteness of spectra of singular Sturm-Liouville problems, Comm. Pure Appl. Math. 12 (1959), 523-542.

[5] Bohner, M., Peterson, A., Dynamic Equations on Time Scales, Birkhäuser, Boston, 2001.

[6] Bohner, M., Peterson, A. (Eds.), Advances in Dynamic Equations on Time Scales, Birkhäuser, Boston, 2003.

[7] Dunford, N., Schwartz, J. T., Linear Operators, Part II: Spectral Theory, Interscience, New York, 1963.

[8] Friedrics, K., Criteria for the discrete character of the spectra of ordinary differential equations, Courant Anniversary Volume, Interscience, New York, 1948.

[9] Friedrics, K., Criteria for discrete spectra, Comm. Pure. Appl. Math. 3 (1950), 439449.

[10] Glazman, I. M., Direct methods of the qualitative spectral analysis of singular differential operators, Israel Program of Scientific Translations, Jerusalem, 1965.

[11] Guseinov, G. Sh., Self-adjoint boundary value problems on time scales and symmetric Green's functions, Turkish J. Math. 29 (4) (2005), 365-380.

[12] Guseinov, G. Sh., An expansion theorem for a Sturm-Liouville operator on semiunbounded time scales, Adv. Dyn. Syst. Appl. 3 (1) (2008), 147-160.

[13] Hilger, S., Analysis on measure chains - a unified approach to continuous and discrete calculus, Results Math. 18 (1-2) (1990), 18-56.

[14] Hinton, D. B., Lewis, R. T., Discrete spectra criteria for singular differential operators with middle terms, Math. Proc. Cambridge Philos. Soc. 77 (1975), 337-347.

[15] Huseynov, A., Weyl's limit point and limit circle for a dynamic systems, in: Dynamical Systems and Methods, Springer, New York, 2012, 215-225. 
[16] Ismagilov, R. S., Conditions for semiboundedness and discreteness of the spectrum for one-dimensional differential equations, Dokl. Akad. Nauk SSSR 140 (1961), 33-36 (Russian).

[17] Jones, M. A., Song, B., Thomas, D. M., Controlling wound healing through debridement, Math. Comput. Modelling 40 (9-10) (2004), 1057-1064.

[18] Kreyszig, E., Introductory Functional Analysis with Applications, Wiley, New York, 1989.

[19] Lakshmikantham, V., Sivasundaram, S., Kaymakcalan, B., Dynamic Systems on Measure Chains, Kluwer Academic Publishers, Dordrecht, 1996.

[20] Molchanov, A. M., Conditions for the discreteness of the spectrum of self-adjoint second-order differential equations, Trudy Moskov. Mat. Obs. 2 (1953), 169-200 (Russian).

[21] Naimark, M. A., Linear Differential Operators, 2nd edition., Nauka, Moscow, 1969, English transl. of 1st edition, Frederick Ungar Publishing Co., New York, 1969.

[22] Rollins, L. W., Criteria for discrete spectrum of singular self-adjoint differential operators, Proc. Amer. Math. Soc. 34 (1972), 195-200.

[23] Rynne, B. P., $L^{2}$ spaces and boundary value problems on time-scales, J. Math. Anal. Appl. 328 (2007), 1217-1236.

[24] Spedding, V., Taming nature's numbers, New Scientist 179 (2003), 28-31.

[25] Thomas, D. M., Vandemuelebroeke, L., Yamaguchi, K., A mathematical evolution model for phytoremediation of metals, Discrete Contin. Dyn. Syst. Ser. B (2) (2005), 411-422.

[26] Weyl, H., Über gewöhnliche Differentialgleichungen mit Singularitäten und die zugehörigen Entwicklungen willkürlicher Funktionen, Math. Ann. 68 (2) (1910), 220269.

Bilender P. Allahverdiev

Department of Mathematics

Süleyman Demirel University

32260 Isparta

Turkey

e-mail: bilenderpasaoglu@sdu.edu.tr

Received August 21, 2017
Hüseyin Tuna

Department of Mathematics

Mehmet Akif Ersoy University

15030 Burdur

Turkey

e-mail: hustuna@gmail.com 